\title{
TITLE:
}

\section{Interstitial lung disease in myositis: clinical subsets, biomarkers, and treatment.}

$\operatorname{AUTHOR}(S)$ :

Mimori, Tsuneyo; Nakashima, Ran; Hosono, Yuji

\section{CITATION:}

Mimori, Tsuneyo ... [et al]. Interstitial lung disease in myositis: clinical subsets, biomarkers, and treatment.. Current rheumatology reports 2012, 14(3): 264-274

ISSUE DATE:

2012-06

URL:

http://hdl.handle.net/2433/156137

\section{RIGHT:}

The final publication is available at www.springerlink.com; This is not the published version. Please cite only the published version.; この論文 は出版社版でありません。引用の際には出版社版をご確認ご利用くだ さい。 
Review article in Current Rheumatology Reports

\section{Interstitial Lung Disease in Myositis: Clinical Subsets, Biomarkers and Treatment}

Tsuneyo Mimori, M.D., Ph.D.*, Ran Nakashima, M.D., Ph.D,, and Yuji Hosono, M.D.

Department of Rheumatology and Clinical Immunology, Graduate School of Medicine, Kyoto University

54 Shogoin-Kawahara-cho, Sakyo-ku, Kyoto 606-8507, Japan

Phone: +81-75-751-4379, FAX: +81-75-751-4338

E-mail: mimorit@kuhp.kyoto-u.ac.jp (T. Mimori)

ranran@kuhp.kyoto-u.ac.jp (R. Nakashima)

hosono25@hotmail.com (Y. Hosono)

${ }^{*}$ Correspondence should be addressed.

\section{Key Words:}

Idiopathic inflammatory myopathy; polymyositis; dermatomyositis; clinically amyopathic dermatomyositis; interstitial lung disease; interstitial pneumonia; NSIP; anti-synthetase antibody; anti-CADM-140 antibody; aminoacyl-transfer RNA synthetase; MDA5; IFIH1; HRCT; KL-6; SP-D; ferritin; glucocorticoid; cyclophosphamide; cyclosporine; tacrolimus; rituximab 


\begin{abstract}
Interstitial lung disease (ILD) is the most frequent organ involvement found in near half of myositis patients, but it reveals various clinical course and therapeutic responsiveness according to the clinical and serological subsets. Autoantibodies, as well as imaging and histopathological studies, are useful for the classification of ILD in myositis and give useful information for predicting the prognosis and determining treatment. Anti-synthetase antibodies are correlated with chronic and recurrent ILD, whereas anti-CADM-140 (MDA5/IFIH1) antibody is a marker of acute progressive ILD in clinically amyopathic dermatomyositis. Serum KL-6, SP-D and ferritin are useful biomarkers for monitoring activity and severity of ILD. As treatment, glucocorticoid is the first-line drug, but additional immunomodulating drugs are also used in refractory patients. In these drugs, cyclophosphamide and calcineurin inhibitors (cyclosporine and tacrolimus) appear to be the key drugs for the treatment of refractory myositis-ILD. Rituximab may become another candidate if these drugs are not effective.
\end{abstract}




\section{Introduction}

Idiopathic inflammatory myositis (IIM), including polymyositis (PM)/ dermatomyositis (DM) is systemic inflammatory disorders which involve not only the muscle and skin but also many organs such as joint, heart and lung. A number of autoantibodies can be detected in sera from PM/DM patients, some of which are specific to PM/DM (known as myositis-specific autoantibodies: MSAs) or myositis overlap syndrome (known as myositis-associated autoantibodies: MAAs). These autoantibodies are closely associated with subsets, complications, reactivity to therapy and prognosis of PM/DM [1].

Interstitial lung disease (ILD) or interstitial pneumonia (IP) is the most common internal organ manifestation that affects the life prognosis of PM/DM patients. Therefore, diagnosis and evaluation of ILD is also very important to determine the treatment strategy when $\mathrm{PM} / \mathrm{DM}$ is diagnosed [2-4].

Recently, two types of MSAs, namely anti-aminoacyl transfer RNA synthetases and anti-CADM-140 (MDA5/IFIH1) antibodies, have been elucidated to be closely associated with ILD in myositis. Moreover, ILD associated with these two antibodies represent completely different clinical subsets each other, providing us useful information for predicting clinical course and prognosis of ILD and for conducting the optimal treatment.

In this article, I will review recent advances and update on the clinical subsets, biomarkers and treatment of ILD associated with PM and DM.

\section{Epidemiologic aspect of ILD in myositis}

The prevalence of ILD in myositis has been reviewed in many myositis cohorts. In earlier era, the lung involvement in PM/DM had considered to be rather rare, as only 5\% was reported in a review by Frazier and Miller in 1974 [5]. However, probably the development and routine use of CT scan enables to detect early interstitial change of the lung, and then the prevalence of ILD has become increased. In the recent cohort studies (single and multicenter), the prevalence of ILD in myositis has reached to near half, ranging from $21 \%$ to $78 \%[6-13]$.

PM/DM patients accompanied ILD have poorer prognosis than those without ILD [9-11]. Prevalence of ILD seems to be similar in both PM and DM, but DM-ILD has obviously more severe course, more refractory to treatment and poorer prognosis than PM-ILD [14-16]. This may be attributed to the presence of anti-CADM-140 antibody, a poor prognostic marker of DM and amyopathic DM, which may be included in cohorts of DM-ILD as discussed later.

\section{Autoantibodies associated with ILD in myositis: Association between clinical and serological subsets of myositis-ILD}


A number of autoantibodies can be detected in sera from IIM patients and are closely associated with clinical subsets of IIM. In these MSAs, anti-aminoacyl transfer RNA synthetases and anti-CADM-140 (MDA5/IFIH1) antibodies have been known to be closely associated with ILD in myositis. However, ILD associated with these two antibodies represent different clinical subsets with different clinical course, prognosis and response to therapy (see Table).

\section{1) Anti-aminoacyl-transfer RNA synthetase antibodies and ILD}

Aminoacyl-transfer RNA synthetases (abbreviated as ARS or synthetase) are the enzymes which catalyze the binding of amino acids to their corresponding transfer RNAs and so there are 20 kinds of synthetases. Among MSAs, anti-synthetase antibodies are found most frequently in PM/DM patients and eight different autoantibodies reacting with different synthetases have been identified so far: anti-Jo-1 (histidyl) [17,18], anti-PL-7 (threonyl) [19], anti-PL-12 (alanyl) [20], anti-EJ (glycyl) [21], anti-OJ (isoleucyl) [22], anti-KS (asparaginyl) [23], anti-Zo (phenylalanyl) [24] and anti-tyrosyl-tRNA synthetase antibodies [25]. With a few exceptions, each patient has only one of these autoantibodies, but patients show similar clinical manifestations, including ILD, myositis, arthritis, fever, Raynaud's phenomenon and mechanic's hand, called 'anti-synthetase syndrome' [26]. ILD is the most frequent extramuscular manifestation that is found in $79-95 \%$ of patients with anti-synthetase antibodies [27-31].

Although anti-synthetase-positive patients show similar clinical manifestations of anti-synthetase syndrome, some detailed clinical studies suggest that there are some differences in clinical manifestations among patients with different anti-synthetase antibodies. Anti-Jo-1 is closely associated with high prevalence of both myositis and ILD, whereas anti-OJ, anti-PL-12 and anti-KS are rather stronger association with ILD than myositis [32-35]. Anti-PL-7 antibody may be associated with PM-scleroderma overlap syndrome as well as ILD [36].

Detailed clinical features of ILD in patients with anti-synthetase antibodies have been described in several reports. The characteristics of ILD in anti-synthetase-positive patients show mostly chronic clinical course but subacute course is also found [37]. In one third to half patients with anti-synthetases, ILD precedes the development of myositis [29,30]. Yoshifuji et al reported the usefulness of anti-synthetase in clinical course prediction of ILD with IIM patients [29]. This retrospective study analyzed 74 patients with myositis in whom 41 had ILD. Any of anti-synthetases were detected in 28\% (21/74) of whole IIM patients. ILD was accompanied in $41(55 \%)$ of 74 IIM patients and anti-synthetases was found in $49 \%$ (21/41) of IIM-ILD patients. Anti-synthetase-positive patients had significantly higher frequency of ILD (95\%) than negative patients (40\%), and ILD of the most positive patients were diagnosed at the same time or before developing myositis. ILD of patients with anti-synthetases showed a better response to initial glucocorticoid therapy 
but revealed significant higher recurrence than those without anti-synthetases. As the result, the 2-year prognosis of pulmonary function was not different between the two groups of each anti-synthetases status. The detection of anti-synthetases may be useful to predict the late-onset myopathy in ILD-preceding patients and to predict the clinical course of ILD in myositis patients.

Anti-/SS-A/Ro antibody is detected in various connective tissue diseases but anti-Ro52 antibody is categorized in one of MAAs. Isolated anti-Ro52 in myositis patients is often correlates with anti-Jo-1 antibody [38-40]. Anti-SS-A/Ro antibody in patients with anti-synthetase syndrome seems to be associated with the development of a more severe ILD [39] and a more frequent association with fibrosis of lung and a less efficacy of immunosuppressive therapy [40].

The production of disease-specific autoantibodies may closely correlate to pathogenic mechanisms of inflammatory myopathy. Patients with antibodies to different synthetases show the same clinical syndrome. This fact strongly suggests that the immune response to molecules with analogous functions leads a similar clinical syndrome. However, the pathophysiologic role of anti-synthetase antibodies in myositis and/or ILD remains to be clarified. There are several reports that suggest a possible pathogenic role of anti-synthetases.

In an animal model, immunized mice with murine Jo-1 antigen generated specific $\mathrm{B}$ and $\mathrm{T}$ cells targeting species-specific epitopes of murine Jo-1 and developed a phenotype consistent with muscle and lung inflammation resembling features of human anti-synthetase syndrome [41]. Sera from anti-Jo-1-positive PM-ILD patients induced a significant effect on the expression of ICAM-1 from human lung endothelial cells [42], although autoantibodies themselves might not be the endothelial cell-activating factor since purified IgG did not induce ICAM-1 expression.

Recent reports demonstrate that certain synthetase molecules (histidyl-, asparaginyl-, and seryl-tRNA synthetases) and their proteolytic fragments have chemokine-like activities against inflammatory cells such as CD4+ and CD8+T cells, and activated monocytes and immature dendritic cells [43]. Mononuclear cells expressing chemokine receptors such as CCR3 and CCR5 infiltrate in muscle tissues of myositis patients but not in normal muscle, supporting the results that histidyl- and asparaginyl-tRNA synthetases activate CCR5+ and CCR3+ cells, respectively [43]. These findings indicate that the liberation of autoantigenic synthetases from damaged tissues may recruit inflammatory mononuclear cells and perpetuate the inflammation process of myositis and ILD, and also induce autoimmune responses to autoantigens.

\section{2) Anti-CADM-140 (MDA5/IFIH1) antibody and ILD}

Amyopathic DM (ADM) and clinically ADM (C-ADM) is defined as the disorder which shows the typical skin manifestations of DM but no or little evidence of clinical myositis [44,45]. It is known that $\mathrm{C}-\mathrm{ADM}$ patients mostly in Asian countries frequently develop life-threatening 
acute progressive ILD [46-50].

Until recently it was thought that MSAs could not be detected in patients with C-ADM and this appeared to be a characteristic feature. In 2005, however, Sato et al reported the identification of a specific autoantibody in C-ADM patients [51]. They screened the sera of 314 patients and controls by ${ }^{35} \mathrm{~S}-\mathrm{methionine-labeled}$ protein immunoprecipitation and immunoblotting techniques using K562 cells, and 8 of 15 patients with C-ADM immunoprecipitated a $140 \mathrm{kD}$ protein. This newly identified autoantibody was named as anti-CADM-140 antibody. In 15 patients with C-ADM, 13 developed ILD and 5 were acute ILD. In the 5 patients with acute ILD, 4 had anti-CADM-140 antibody. Later, the presence of anti-CADM-140 and the association with C-ADM-ILD have been confirmed in worldwide. The antibody can be detected exclusively in DM (11-26\%) or C-ADM (50-73\%) [52-56].

Nakashima et al reported the characteristics of anti-CADM-140-positive patients [52]. In screening of 192 patients with various CTD, 13 revealed to be positive with anti-CADM-140 antibody. All anti-CADM-140-positive patients were DM either typical DM ( 2 cases) or C-ADM (11 cases), and $9(69 \%)$ patients had fever $>38^{\circ} \mathrm{C}$, while 12 patients $(92 \%)$ had ILD and 7 (54\%) developed acute progressive ILD. Life prognosis was significantly poorer in anti-CADM-140-positive patients than in anti-CADM-140-negative DM patients, and $6(46 \%)$ died of respiratory failure within 6 months from the onset of disease. Interestingly, the serum ferritin concentrations in 11 of anti-CADM-140-positive patients were already elevated within one month of their admission with significantly high frequency in comparison with anti-CADM-negative DM patients ( 85 vs. $33 \%$; $=0.005$ ). Moreover, the serum ferritin level correlated to the activity of ILD in anti-CADM-140-positive patients.

ILD in anti-CADM-140-positive patients was correlated abnormalities in serum hepatobiliary enzymes and interleukin-18, which worsened in accordance with ILD and ferritin levels $[57,58]$. Thus, the anti-CADM-140 antibody appeared to be associated with macrophage activation syndrome developed in C-ADM and intractable acute ILD.

The target autoantigen of anti-CADM-140 antibody was identifies as melanoma differentiation-associated gene 5 (MDA5), also known as interferon induced with helicase $\mathrm{C}$ domain protein 1 (IFIH1) [52,59]. MDA5/IFIH1 is one of the retinoic acid-inducible gene-I (RIG-I)-like receptors, which are involved in the recognition of viral RNAs and play an important role in innate immune responses. RIG-I and MDA5/IFIH1 are able to interact with viral RNA and mediate signaling pathways leading to the expression of type I interferon and inflammatory cytokines.

Finding MDA5/IFIH1 as the autoantigen specifically recognized by one of DM-specific autoantibodies is strikingly interested, because many reports have suggested the possible association between myositis and viral infections [60-62], in particular Coxsackie B virus belonging to the picornaviruses that are targeted by MDA5/IFIH1. To increase our understanding of the pathophysiology of acute ILD accompanied with C-ADM and to develop more effective therapy, whether anti-CADM-140 antibody and its target antigen MDA5/IFIH1 have pathogenic roles in ILD and C-ADM should be elucidated. 


\section{Radiological and histopathological findings of ILD in myositis}

In histopathological analysis by VATS or TBLB, nonspecific interstitial pneumonia (NSIP) is the most frequent histological pattern of ILD in myositis, but usual interstitial pneumonia (UIP), organizing pneumonia (OP) and diffuse alveolar damage (DAD) can also be found though less frequent [14,63-65]. These histological findings are partially but not always associated with the underlying disease (PM or DM), clinical course (acute or chronic) and autoantibody profiles. While NSIP is widely found in both PM and DM, UIP is associated with chronic ILD (both PM and DM), and DAD is characteristically found in acute refractory ILD in DM and C-ADM. OP shows a good response to glucocorticoid therapy.

High resolution computed tomography (HRCT) scanning of chest is a most sensitive technique to detect ILD, and provide information for prognosis and response of treatment.

Typical HRCT findings in myositis-associated ILD include ground glass opacities (GGO), micronodules, linear and reticular opacities, peribronchovascular or subpleural consolidation, irregularity of interface, and traction bronchiectasis [15,46,65,66] (see Figure). These findings are compatible with NSIP. Honeycombing pattern suggesting UIP is also found in ILD-myositis but with lower frequency.

Recent studies suggest characteristics of HRCT findings in two myositis/ILD-associated autoantibodies. Watanabe et al reported the HRCT and pathological findings in 13 ILD cases with anti-synthetase syndrome but without clinical myositis [67]. Pleural irregularities and/or prominent interlobular septa, GGO, reticulation and traction bronchiectasis were the major findings, while honeycombing was not found in any case. Although most of these cases showed histological NSIP if lung biopsies were applied, even in cases with pathological diagnosis of UIP, HRCT findings were not compatible with a typical UIP pattern showing honeycombing.

Tanizawa et al described HRCT findings of ILD in DM/C-ADM with anti-CADM-140 antibody [68]. GGO, non-septal linear or plate-like opacity and consolidation were the common findings in ILD of both antibody-positive and negative DM. However, intralobular reticular opacities (abnormal thickening of intralobular interstitial tissues) found in the antibody-negative ILD was significantly less in the positive ILD. Moreover, lower consolidation/GGO pattern (lower peripheral or peribronchovascular consolidation or GGO) and random GGO pattern (random peripheral GGO) were mainly found in anti-CADM-140-positive patients, whereas lower reticulation pattern (lower peripheral or peribronchovascular reticulation) was the main finding of anti-CADM-140-negative patients. 
The latter HRCT pattern is compatible with NSIP and likely to that of anti-synthetase-positive ILD, while three patients who were negative for both anti-CADM-140 and anti-synthetases showed lower reticulation pattern as well. Thus, HRCT of anti-CADM-140- positive DM/C-ADM-ILD is characterized by lower consolidation/GGO and random GGO patterns and the absence of intralobular reticular opacities, which are different from typical NSIP patterns (see Figure).

Lower reticulation pattern is consistent with NSIP and likely to that of anti-synthetase-positive ILD. On the other hand, lower consolidation/GGO and random GGO patterns in anti-CADM-140-positive ILD are hard to interpret because the pathological approach is usually difficult in early stage of the disease. Lower consolidation/GGO may represent OP or localized DAD [68]. These findings and the absence of lower reticulation and intralobular reticular opacities in anti-CADM-140-positive ILD may suggest a lower prevalence of pathological NSIP.

\section{Biomarkers of ILD in myositis}

There are no specific serum biomarkers for myositis-associated ILD. Nevertheless, certain serum markers predicting activity of ILD should be necessary, since a correlation is often not recognized between activities of myositis and ILD. HRCT of lung is the most valuable and sensitive method to detect and predict the activity of ILD, but frequent examination should avoid because of the risk for high radiation exposure by CT. Several biomarkers that may reflect inflammatory activity of lung have been utilized.

\section{1) KL-6 and SP-D}

KL-6 (Krebs von den Lungen-6) is a mucin-like glycoprotein expressed in type II alveolar epithelial cells. Since serum level of KL-6 increases by reflecting hyper-expression of KL-6 producing cells and alveolar injury in ILD, its detection is useful as a serologic biomarker for diagnosis and monitoring of ILD. Several studies suggested the usefulness of KL-6 in disease activity, therapeutic response and prognosis of myositis-ILD [69-74]. Satoh et al demonstrated that high serum level (>1000 U/ml) of KL-6 was a poor prognostic factor in 152 idiopathic IP and 67 connective tissue disease-associated ILD including $16 \mathrm{PM} / \mathrm{DM}$ patients before treatment [72].

SP-D (Surfactant protein-D) is a lung-specific surfactant lipoprotein secreted from type II alveolar epithelial cell. Serum level of SP-D reflects the activity of certain lung diseases such as ILD and pulmonary alveolar proteinosis [71,73]. Comparing with KL-6, SP-D appears to have same specificity but lower sensitivity [71].

\section{2) Ferritin}


Recent studies suggest serum ferritin level as a marker for severity of acute progressive ILD in $\mathrm{DM}$ and $\mathrm{C}-\mathrm{ADM}$ patients $[52,57,75,76]$. The serum ferritin concentrations in anti-CADM-140-positive patients are already elevated from early stage of the disease with significantly high frequency in comparison with antibody-negative DM patients even if ILD is not worsened [52]. Moreover, the serum ferritin concentration correlates to the activity of ILD in anti-CADM-140-positive patients.

Gono et al showed that serum ferritin level before initial treatment in acute or subacute ILD in DM patients was significantly higher (mean $790 \mathrm{ng} / \mathrm{ml}$ ) than in chronic ILD (188 $\mathrm{ng} / \mathrm{ml}$ ) and non-ILD patients (160 ng/ml) [75]. Patients with ferritin level more than 1500 $\mathrm{ng} / \mathrm{ml}$ showed significantly poorer prognosis than those with less than $1500 \mathrm{ng} / \mathrm{ml}$. They also reported that serum ferritin was significantly elevated in patients with anti-CADM-140 (MDA5)-positive acute ILD and correlated with prognosis and disease activity $[57,76]$.

\section{3) Titers of autoantibodies}

It is not clarified whether myositis-specific autoantibodies are directly involved in pathophysiologic mechanisms of myositis and ILD. Correlation between titers of such autoantibodies and activity of myositis or ILD is controversial. There are several reports that anti-Jo-1 antibody titers correlated with activity of myositis as well as lung [77], and anti-CADM-140 titer disappeared after intensive treatment of ILD in ADM patients [78]. However, these changes of antibody titers are modest, and further studies will be necessary for conclusion.

\section{Treatment}

Regarding the therapy of ILD in myositis, glucocorticoids are the empirical first-line drug, and additional immunosuppressive agents are often used as the second-line drugs in cases of refractory disease. However, these therapies are not based on high levels of evidence (i.e. randomized controlled trials) but based on long-term experiences, retrospective studies and small-scale case series or case reports. Since ILD is a progressive and fatal disease and glucocorticoids have been established as the standard therapy, it is difficult to conduct placebo-controlled prospective trials.

\section{1) Glucocorticoids (GC)}

Oral high-dose GC (more than $1 \mathrm{mg} / \mathrm{kg} /$ day of predonisone) or pulse therapy of methylprednisolone (1000mg iv for 3days) is the first-line therapy of ILD in myositis. Roughly half of ILD in myositis is effective to the initial GC therapy [14,29,37,79]. However, acute ILD, especially rapid progressive ILD in anti-CADM-140-positive DM/C-ADM is usually not responsive to GC alone [51-56].

Fujisawa et al reported 28 ILD with myositis (16 PM and $12 \mathrm{DM}$ ) and their difference in 
efficacy of treatment [14]. GC alone achieved a favorable response in 6 patients $(37.5 \%)$ with PM-ILD but in only one (8.3\%) with DM-ILD. Overall 2.5-year survival in DM-ILD was $58 \%$ and 5-year survival in PM-ILD was $81 \%$. In the similar study by Nawata et al, when ILD was classified into two groups according to serum creatine kinase (CK) levels (high CK or normal CK), the normal CK group showed significantly more resistant to GC therapy and poorer prognosis than those with high CK (one-year survival 31\% vs. 89\%) [79].

Although these studies have not reported the autoantibody status, GC-refractory ILD with DM (not PM) or normal CK patients may represent the characteristic features of patients with anti-CADM-140 antibody, who have reported to have treatment resistance and poor prognosis. It has been obvious that the prognosis of initial GC-resistant ILD patients is not improved even if immunosuppressive drugs add after exacerbation of respiratory symptoms. The efficacy of GC as the initial therapy of ILD is limited, nevertheless GC is still the mainstay of therapy.

\section{2) Cyclophosphamide (CYC)}

CYC, oral or intravenous pulse (IVCYC), is commonly used in acute or refractory ILD, and has demonstrated its efficacy in several case series and small-scale open label trials [7,80-82]. Yamasaki et al reported 17 cases of refractory ILD in myositis who treated with IVCYC $\left(300-800 \mathrm{mg} / \mathrm{m}^{2}\right.$ at least 6 times every 4 weeks) and showed significant improvement in dyspnea, pulmonary function and HRCT findings [82].

CYC is also used in combination with other immunosuppressive drugs in refractory ILD [83,84]. Kameda et al reported the efficacy of IVCYC in combination with GC and cyclosporine in $10 \mathrm{DM}$ patients with acute ILD [84]. Although 5 patients who received the combination therapy died of respiratory failure within 3 month, the mortality rate tended to become lower than in the historical control in which 9 out of 12 patients died despite high dose GC with or without a choice of CYC, cyclosporine or azathioprine.

\section{3) Calcineurin inhibitors}

Calcineurin inhibitors such as cyclosporine and tacrolimus targeting activated $\mathrm{T}$ cells by inhibiting calcineurin and nuclear translocation of NF-AT may become the cornerstone for the treatment of ILD in myositis.

\section{a. Cyclosporine (CSP)}

There are several retrospective or open-label studies to analyze the efficacy of CSP in PM/DM-ILD [14,37,85,86,79]. Nagasaka et al reported a multicenter retrospective analysis of 38 cases with acute ILD in PM/DM [86]. In this study, ILD in PM (9 cases) and chronic ILD in DM (5 cases) showed good efficacy of CSP and good prognosis, whereas patients with acute IP in DM showed poor response and poor prognosis (7 out of 17 dead). Moreover, in a further analysis of 32 cases with acute DM-ILD, 9 out of 13 cases starting CSP within 2 weeks from initial GC were survived (survival rate 69\%), whereas all 17 cases receiving only GC more than 2 weeks as the initial therapy died within 9 months from the therapy. In the 
retrospective study by Kotani et al analyzing the efficacy of CSP in 16 DM cases with acute or subacute ILD, 9 cases treated initially with GC and CSP (mean interval 3.8 days) appeared to show good prognosis (only one dead), whereas 4 out of 7 cases (57\%) in whom CSP was added when initial GC not effective (mean interval 20.0 days) died of respiratory failure related to ILD [87].

Monitoring of serum CSP concentration is important for achieving maximal efficacy and for reducing toxicity. Trough level (C0) and 2-hour post-dose blood concentration (C2) are correlated with the therapeutic effects [88,89]. They also suggest that once daily preprandial administration of CSP, rather than twice daily, may be beneficial in DM patients with progressive ILD [89]. These studies, although small scaled retrospective analyses, suggest the effectiveness of early intervention and tight control by CSP in combination with GC in the treatment of refractory myositis-ILD.

\section{b. Tacrolimus (TAC)}

TAC is another calcineurin inhibitor and has 100 -fold more potent to inhibit $\mathrm{T}$ cell activity comparing with CSP. Recently TAC has been utilized in refractory ILD in myositis patients as well as CSP. Several case series and retrospective studies show the efficacy and tolerability of TAC in patients with PM/DM-ILD refractory to CSP [86,90-92]. TAC appears to be more effective in ILD of anti-synthetase syndrome [91,92]. Wilkes et al retrospectively analyzed 13 anti-synthetase-positive patients treated with TAC and showed its efficacy both for refractory ILD and myositis being well tolerated [92].

\section{4) Rituximab}

Rituximab is a biologic agent of chimeric monoclonal anti-CD20 antibody that targets B cell. Recently its potential usefulness has been demonstrated in various autoimmune diseases, and several case reports and case series of rituximab have also been reported in myositis-ILD [93-95]. Sem et al reported 11 refractory ILD in anti-synthetase syndrome patients treated with rituximab and showed that rituximab stabilized and/or improved the ILD in 7 of 11 patients [93]. Rituximab reduced the serum titer of anti-Jo-1 antibody but the effect was modest.

\section{5) Other immunomodulating therapies}

\section{a. Methotrexate (MTX)}

Although MTX, inhibitor of folic acid and purine metabolisms, is widely used in treatment of refractory PM/DM, there is no obvious evidence on myositis-ILD. The use of MTX in ILD is controversial because there are patients that respond favorably to this treatment but the risk for idiosyncratic drug-induced ILD is reported on the other hand. Particular care should be taken if using MTX for ILD in myositis.

\section{b. Azathioprine (AZT)}

AZT has been widely used in myositis and ILD as the second-line immunomodulating drug, but its efficacy is modest. AZT may be useful as a maintenance therapy for the control of 
ILD after CYC.

\section{c. Mycophenolate mofetil (MMF)}

In a few case series studies, the potential efficacy of MMF has been shown in stabilization of progressive ILD and reducing GC dose in ILD patients with connective tissue diseases including PM/DM [96-97].

\section{d. Intravenous immunoglobulin (IVIG)}

The efficacy of IVIG has been demonstrated in muscular symptoms of refractory DM and PM, but the usefulness in ILD associated with myositis is uncertain. One case series of 5 patients with severe ILD reported a potential usefulness as a salvage therapy [98].

\section{e. Anti-cytokine biologics}

The use of anti-TNF [99,100] and other biologics (including tocilizumab [101] and anakinra [102]) have been reported in some case reports and case series and in a randomized pilot trial [100]. However, all of them focused on severe or refractory myopathy but not on ILD. They appear to be sometimes effective on myositis but some reports suggest no effect or even worsening muscle symptoms [99]. Their efficacy on ILD is not discussed. Experience of anti-cytokine therapies on myositis-ILD should be collected and carefully investigated.

\section{Conclusion}

ILD is the most frequent extra-muscular organ involvement and the most important prognostic factor of IIM, but it reveals various clinical course and therapeutic responsiveness according to the clinical and serological subsets. Autoantibodies, as well as imaging and histopathological studies, are useful for the classification of ILD in myositis, and give us useful information for predicting the prognosis and determining therapeutic strategy. Routine examination of anti-CADM-140 antibody and anti-synthetase antibodies except for anti-Jo-1 are not available so far, since these antibodies can only be detected by complicated immunoprecipitation techniques. Quantitative methods to detect these MSAs are now developing and will be available in near future.

As treatment of ILD in myositis, GC is still the first-line drug and various immunomodulating drugs are also frequently used in refractory patients. In these drugs, CYC (especially IVCYC) and calcineurin inhibitors (CSP and TAC) appear to be the key drugs for the treatment of refractory ILD in myositis. Rituximab may be another candidate if these drugs are not effective. However, there are no large-scale, randomized clinical trials to guarantee the efficacy and safety of these drugs. Although it may be difficult to conduct placebo-controlled trials in such progressive and fatal condition, the construction of evidences should be necessary to achieve the best management of intractable disease like ILD.

Acknowledgment This work was supported by a grant-in-aid for scientific research from 
the Japan Society for the Promotion of Science, and a grant for intractable diseases from the Ministry of Health, Labor and Welfare, Japan.

Disclosure There is no conflict of interest to any authors of this article. 


\section{References}

Papers of particular interest, published recently, have been highlighted as:

- Of inportance

- Of major importance

1. Nakashima, R., Mimori, T: Clinical and pathophysiological significance of myositis-specific and myositis-associated autoantibodies. Int J Clin Rheumatol 2010, 5: $523-536$.

2. @Saketkoo LA, Ascherman DP, Cottin V, et al.: Interstitial Lung Disease in Idiopathic Inflammatory Myopathy. Curr Rheumatol Rev 2010, 6:108-119. A good review article of ILD in IIM focusing on the pathogenesis, clinical manifestation, diagnosis and disease monitoring, and therapeutics.

3. Connors GR, Christopher-Stine L, Oddis CV, et al.: Interstitial lung disease associated with the idiopathic inflammatory myopathies. What progress has been made in the past 35 years? Chest 2010, 138:1464-1474. A good review article of ILD in IIM focusing on pathogenesis, diagnosis and current therapeutic approarches.

4. Labirua A, Lundberg IE: Interstitial lung disease and idiopathic inflammatory myopathies: progress and pitfalls. Curr Opin Rheumatol. 2010, 22:633-638. A good review article describing recent findings regarding ILD in IIM, focusing on the phenotypes of ILD, autoantibodies, pathogenesis and treatment.

5. Frazier AR, Miller RD: Interstitial pneumonitis in association with polymyositis and dermatomyositis. Chest 1974, 65:403-407.

6. Marie I, Hachulla E, Chérin P, et al.: Interstitial lung disease in polymyositis and dermatomyositis . Arthritis Rheum 2002, 47: 614-622.

7. Schnabel A, Reuter M, Biederer J, et al.: Interstitial lung disease in polymyositis and dermatomyositis: clinical course and response to treatment. Semin Arthritis Rheum 2003, $32: 273-284$.

8. Fathi M, Dastmalchi M, Rasmussen E, et al. et al. Interstitial lung disease, a common manifestation of newly diagnosed polymyositis and dermatomyositis. Ann Rheum Dis 2004, 63: 297-301.

9. Kang EH, Lee EB, Shin KC, et al.: Interstitial lung disease in patients with polymyositis, dermatomyositis and amyopathic dermatomyositis. Rheumatology (Oxford) 2005, $44: 1282-1286$.

10. Ye S, Chen XX, Lu XY, et al. Adult clinically amyopathic dermatomyositis with rapid progressive interstitial lung disease: a retrospective cohort study . Clin Rheumatol 2007, 26: $1647-1654$.

11. Won Huh J, Soon Kim D, Keun Lee C, et al.: Two distinct clinical types of interstitial lung disease associated with polymyositis-dermatomyositis. Respir Med 2007, 101: 1761-1769. 
12. Fathi M, Vikgren J, Boijsen M, et al.: Interstitial lung disease in polymyositis and dermatomyositis: longitudinal evaluation by pulmonary function and radiology. Arthritis Rheum 2008,59:677-685.

13. Chen IJ, Jan Wu YJ, Lin CW, et al.: Interstitial lung disease in polymyositis and dermatomyositis. Clin Rheumatol. 2009, 28(6):639-646.

14. Fujisawa T, Suda T, Nakamura Y, et al.: Differences in clinical features and prognosis of interstitial lung diseases between polymyositis and dermatomyositis. J Rheumatol 2005, 32:58-64.

15. Hayashi S, Tanaka M, Kobayashi H, et al.: High-resolution computed tomography characterization of interstitial lung diseases in polymyositis/dermatomyositis. $\mathrm{J}$ Rheumatol 2008, 35:260-269.

16. -Yamasaki Y, Yamada H, Ohkubo M, et al.: Longterm survival and associated risk factors in patients with adult-onset idiopathic inflammatory myopathies and amyopathic dermatomyositis: experience in a single institute in Japan. J Rheumatol 2011, 38:1636-1643. Erratum in: J Rheumatol 2011, 38:1815-1816. This retrospective study analyses the lomgterm-suvival and associated risk factors of 197 IIM patients, and suggest that ILD is the main cause of death in DM and $C$ - $A D M$.

17. Nishikai M, Reichlin M: Heterogeneity of precipitating antibodies in polymyositis and dermatomyositis: Characterization of the Jo-1 antibody system. Arthritis Rheum 1980, 23:881-888.

18. Mathews MB, Bernstein RM: Myositis autoantibody inhibits histidyl-tRNA synthetase: A model for autoimmunity. Nature 1983, 304:177-179.

19. Mathews MB, Reichlin M, Hughes GRV, et al: Anti-threonyl-tRNA synthetase, a second myositis-related autoantibody. J Exp Med 1984, 160:420-434.

20. Bunn CC, Bernstein RM, Mathews MB: Autoantibodies against alanyl-tRNA synthetase and tRNAala coexist and are associated with myositis. J Exp Med 1986, 163:1281-1291.

21. Targoff IN, Trieu EP, Plotz PH, et al: Antibodies to glycyl-transfer RNA synthetase in patients with myositis and interstitial lung disease. Arthritis Rheum 1992, 35:821-830.

22. Targoff IN, Trieu EP, Miller FW: Reaction of anti-OJ autoantibodies with components of the multi-enzyme complex of aminoacyl-tRNA synthetases in addition to isoleucyl-tRNA synthetase. J Clin Invest 1993, 91:2556-2564.

23. Hirakata M, Suwa A, Nagai S, et al.: Anti-KS: identification of autoantibodies to asparaginyl-transfer RNA synthetase associated with interstitial lung disease. $\mathrm{J}$ Immunol 1999, 162:2315-2320.

24. Betteridge Z, Gunawardena H, North J, et al.: Anti-synthetase syndrome: a new autoantibody to phenylalanyl transfer RNA synthetase (anti-Zo) associated with polymyositis and interstitial pneumonia. Rheumatology (Oxford) 2007, 46:1005-1008.

25. Hashish L, Trieu EP, Sadanandan P, et al: Identification of autoantibodies to tyrosyl-tRNA synthetase in dermatomyositis with features consistent with anti-synthetase syndrome (abstract). Arthritis Rheum 2005, 52:S312. 
26. Targoff IN: Immune manifestation of inflammatory muscle disease. Rheum Dis Clin North Am 1994, 20:857-880.

27. Yoshida S, Akizuki M, Mimori T, et al.: The precipitating antibody to an acidic nuclear protein antigen, the Jo-1, in connective tissue diseases. A marker for a subset of polymyositis with interstitial pulmonary fibrosis. Arthritis Rheum 1983, 26: 604-611.

28. Marguerie C, Bunn CC, Beynon HL, et al.: Polymyositis, pulmonary fibrosis and autoantibodies to aminoacyl-tRNA synthetase enzymes. Q J Med 1990, 77: 1019-1038

29. Yoshifuji H, Fujii T, Kobayashi S, et al.: Anti-aminoacyl-tRNA synthetase antibodies in clinical course prediction of interstitial lung disease complicated with idiopathic inflammatory myopathies. Autoimmunity 2006, 39:233-241.

30. Matsushita T, Hasegawa M, Fujimoto M, et al.: Clinical evaluation of anti-aminoacyl tRNA synthetase antibodies in Japanese patients with dermatomyositis. J Rheumatol 2007, 34: 1012-1018.

31. @Richards TJ, Eggebeen A, Gibson K, et al.: Characterization and peripheral blood biomarker assessment of anti-Jo-1 antibody-positive interstitial lung disease. Arthritis Rheum 2009, 60: 2183-2192. 90 patients with anti-Jo-1 antibody were analyzed, and $86 \%$ was associated with ILD. A significant association between anti-Jo-1-positive ILD and elevated levels of interferon- $\gamma$-inducible chemokines CXCL9 and CXCL1O was revealed.

32. Hirakata M, Suwa A, Takada T, et al.: Clinical and immunogenetic features of patients with autoantibodies to asparaginyl-transfer RNA synthetase. Arthritis Rheum 2007, 56: 1295-303.

33. Sato S, Kuwana M, Hirakata M: Clinical characteristics of Japanese patients with anti-OJ (anti-isoleucyl-tRNA synthetase) autoantibodies. Rheumatology (Oxford) 2007, 46:842-845.

34. -Kalluri M, Sahn SA, Oddis CV, et al.: Clinical profile of anti-PL-12 autoantibody. Cohort study and review of the literature. Chest 2009, 135: 1550-1556. 31 patients with anti-PL-12 antibody was analysed, and anti-PL-12 was strongly associated with the presence of ILD, but less with myositis and arthritis.

35. Fischer A, Swigris JJ, du Bois RM, et al.: Anti-synthetase syndrome in ANA and anti-Jo-1 negative patients presenting with idiopathic interstitial pneumonia. Respir Med 2009, 103:1719-1724.

36. Sato S, Hirakata M, Kuwana M, et al.: Clinical characteristics of Japanese patients with anti-PL-7 (anti-threonyl-tRNA synthetase) autoantibodies. Clin Exp Rheumatol 2005, 23:609-615.

37. @Koreeda Y, Higashimoto I, Yamamoto M, et al.: Clinical and pathological findings of interstitial lung disease patients with anti-aminoacyl-tRNA synthetase autoantibodies. Intern Med 2010, 49:361-369. Clinicopathological characteristics of ILD in 14 patients with anti-synthetase syndrome is reviewed. Chronic clinical course, lung-base GGO with volume loss, NSIP pattern and good response to CS are their characteristic features. 
38. Rutjes SA, Vree Egberts WT, Jongen P, et al.: Anti-Ro52 antibodies frequently co-occur with anti-Jo-1 antibodies in sera from patients with idiopathic inflammatory myopathy. Clin Exp Immunol 1997, 109: 32-40.

39. La Corte R, Lo Mo Naco A, Locaputo A, et al.: In patients with antisynthetase syndrome the occurrence of anti-Ro/SSA antibodies causes a more severe interstitial lung disease. Autoimmunity 2006,39: 249-253.

40. Vancsa A, Csipo I, Nemeth J, et al.: Characteristics of interstitial lung disease in SS-A positive/Jo-1 positive inflammatory myopathy patients. Rheumatol Int 2009, 29:989-994.

41. Katsumata Y, Ridgway WM, Oriss T, et al.: Species-specific immune responses generated by histidyl-tRNA synthetase immunization are associated with muscle and lung inflammation. J Autoimmun. 2007, 29: 174-186.

42. - Barbasso Helmers S, Englund P, Engström M, et al.: Sera from anti-Jo-1-positive patients with polymyositis and interstitial lung disease induce expression of intercellular adhesion molecule 1 in human lung endothelial cells. Arthritis Rheum 2009, 60:2524-2530. Erratum in: Arthritis Rheum 2009, 60:2746. Sera from PM-ILD with anti-Jo-1 antibody (but might not with antibody itself) showed a strong effect on the expression of ICAM-1 from endotherial cells from human lung.

43. Howard OM, Dong HF, Yang D, et al.: Histidyl-tRNA synthetase and asparaginyl-tRNA synthetase, autoantigens in myositis, activate chemokine receptors on $\mathrm{T}$ lymphocytes and immature dendritic cells. J Exp Med 2002, 196:781-791.

44. Euwer RL, Sontheimer RD: Amyopathic dermatomyositis: a review. J Invest Dermatol 1993, 100:124S-127S.

45. Sontheimer RD: Would a new name hasten the acceptance of amyopathic dermatomyositis (dermatomyositis sine myositis) as a distinctive subset within the idiopathic inflammatory dermatomyopathies spectrum of clinical illness? J Am Acad Dermatol 2002, 46:626-636.

46. Cottin V, Thivolet-Béjui F, Reynaud-Gaubert M, et al.: Interstitial lung disease in amyopathic dermatomyositis, dermatomyositis and polymyositis. Eur Respir J 2003, 22:245-250.

47. Yokoyama T, Sakamoto T, Shida N, et al.: Fatal rapidly progressive interstitial pneumonitis associated with amyopathic dermatomyositis and CD8 T lymphocytes. J Intensive Care Med 2005, 20:160-163.

48. Suda T, Fujisawa T, Enomoto N, et al.: Interstitial lung diseases associated with amyopathic dermatomyositis. Eur Respir J 2006, 28: 1005-1012 .

49. Gerami P, Schope JM, McDonald L, et al.: A systematic review of adult-onset clinically amyopathic dermatomyositis (dermatomyositis sine myositis): a missing link within the spectrum of the idiopathic inflammatory myopathies. J Am Acad Dermatol 2006, 54:597-613.

50. Mukae H, Ishimoto H, Sakamoto N, et al.: Clinical differences between interstitial lung disease associated with clinically amyopathic dermatomyositis and classic 
dermatomyositis. Chest 2009, 136:1341-1347.

51. Sato S, Hirakata M, Kuwana M, et al: Autoantibodies to a 140-kd polypeptide, CADM-140, in Japanese patients with clinically amyopathic dermatomyositis. Arthritis Rheum 2005, 52:1571-1576.

52. ๑ Nakashima R, Imura Y, Kobayashi S, et al.: The RIG-I-like receptor IFIH1/MDA5 is a dermatomyositis-specific autoantigen identified by the anti-CADM-140 antibody. Rheumatology (Oxford) 2010, 49: 433-440. In this article, the target autoantigen recognized by anti-CADM-140 antibody was identifies as IFIH1/MDA5, and at first the association between anti-CADM-140 positive patients and hyperferritinemia was described.

53. -Hoshino K, Muro Y, Sugiura K, Tomita Y, Nakashima R, Mimori T: Anti-MDA5 and anti-TIF $1 \gamma$ antibodies have clinical significance for patients with dermatomyositis. Rheumatology (Oxford) 2010, 49:1726-33. Anti-CADM-140 (MDA5) and anti-TIF1- $\gamma$ antibodies were detected by a new method immunoprecipitating with biotinylated recombinant MDA5 and TIF1- $\gamma .26 \%$ and $65 \%$ were positive for anti-MDA5 in DM and $C$-ADM, respectively. $95 \%$ of the antibody-positive patients had ILD.

54. Kang EH, Nakashima R, Mimori T, et al.: Anti-140-kDa polypeptide antibody is primarily associated with rapidly progressive interstitial lung disease independent of clinically amyopathic dermatomyositis. BMC Musculoskelet Disord. 2010, 11(1):223.

55. Fiorentino D, Chung L, Zwerner J, et al.: The mucocutaneous and systemic phenotype of dermatomyositis patients with antibodies to MDA5 (CADM-140): a retrospective study. $J$ Am Acad Dermatol 2011, 65:25-34.

56. Hamaguchi Y, Kuwana M, Hoshino K, et al: Clinical correlations with dermatomyositis-specific autoantibodies in adult Japanese patients with dermatomyositis: a multicenter cross-sectional study. Arch Dermatol 2011, 147:391-398.

57. ๑ Gono T, Kawaguchi Y, Satoh T, et al.: Clinical manifestation and prognostic factor in anti-melanoma differentiation-associated gene 5 antibody-associated interstitial lung disease as a complication of dermatomyositis. Rheumatology (Oxford) 2010, 49:1713-1719. In this article, serum ferritin level was reported to be significantly high in the subset with anti-CADM-140 (MDA5) antibody, correlate with disease activity and predict prognosis.

58. Gono T, Kawaguchi Y, Sugiura T, et al: Interleukin-18 is a key mediator in dermatomyositis: potential contribution to development of interstitial lung disease. Rheumatology (Oxford). 2010, 49:1878-1881.

59. - Sato S, Hoshino K, Satoh T, et al.: RNA helicase encoded by melanoma differentiation-associated gene 5 is a major autoantigen in patients with clinically amyopathic dermatomyositis: Association with rapidly progressive interstitial lung disease. Arthritis Rheum 2009, 60: 2193-2200. An important article at first identifying the target autoantigen recognized by anti-CADM-140 antibody to be MDA5.

60. Christensen ML, Pachman LM, Schneiderman R, Patel, et al.: Prevalence of Coxsackie B 
virus antibodies in patients with juvenile dermatomyositis. Arthritis Rheum 1986, 29: 1365-1370.

61. Bowles NE, Dubowitz V, Sewry CA et al.: Dermatomyositis, polymyositis, and Coxsackie-B-virus infection. Lancet 1987, 1: 1004-1007.

62. Chevrel G, Calvet A, Belin V, et al: Dermatomyositis associated with the presence of parvovirus B19 DNA in muscle. Rheumatology (Oxford) 2000, 39:1037-1039.

63. Douglas WW, Tazelaar HD, Hartman TE, et al.: Polymyositis-dermatomyositisassociated interstitial lung disease. Am J Respir Crit Care Med 2001, 164:1182-1185.

64. Kalluri M, Oddis CV: Pulmonary manifestations of the idiopathic inflammatory myopathies. Clin Chest Med 2010, 31:501-512.

65. Daimon T, Johkoh T, Honda O, et al: Nonspecific interstitial pneumonia associated with collagen vascular disease: analysis of $\mathrm{CT}$ features to distinguish the various types. Intern Med 2009, 48:753-761.

66. Mino M, Noma S, Taguchi Y, et al.: Pulmonary involvement in polymyositis and dermatomyositis: sequential evaluation with CT. AJR Am J Roentgenol 1997, 169:83-87.

67. -Watanabe K, Handa T, Tanizawa K, at al.: Detection of antisynthetase syndrome in patients with idiopathic interstitial pneumonias. Respir Med 2011, 105:1238-1247. Anti-synthetase antibodies were detected in13 patients (6.6\%) from 198 idiopathic interstitial pneumonia. On HRCT, GGO and traction bronchiectasis were the major findings in these patients.

68. ๑๐anizawa K, Handa T, Nakashima R, et al.: HRCT features of interstitial lung disease in dermatomyositis with anti-CADM-140 antibody. Resp Med 2011, 105:1380-1387. HRCT pattern of anti-CADM-140-positive ILD was described. Lower consolidation or GGO pattern and random GGO pattern but the absence of intralobular reticular opacities were the characteristic HRCT findings of these patients.

69. Bandoh S, Fujita J, Ohtsuki Y, et al.: Sequential changes of KL-6 in sera of patients with interstitial pneumonia associated with polymyositis/dermatomyositis. Ann Rheum Dis 2000, 59: 257-262.

70. Kubo M, Ihn H, Yamane K, et al.: Serum KL-6 in adult patients with polymyositis and dermatomyositis. Rheumatology (Oxford) 2000, 39: 632-636.

71. Ohnishi H, Yokoyama A, Kondo K, et al: Comparative study of KL-6, surfactant protein-A, surfactant protein-D, and monocyte chemoattractant protein-1 as serum markers for interstitial lung diseases. Am J Respir Crit Care Med 2002, 165:378-381.

72. Satoh H, Kurishima K, Ishikawa H, et al.: Increased levels of KL-6 and subsequent mortality in patients with interstitial lung diseases. J Intern Med 2006, 260:429-434.

73. Kumánovics G, Minier T, Radics J, Pálinkás L, et al.: Comprehensive investigation of novel serum markers of pulmonary fibrosis associated with systemic sclerosis and dermato/polymyositis. Clin Exp Rheumatol 2008, 26: 414-420.

74. Fathi M, Barbasso Helmers S, Lundberg IE: KL-6: a serological biomarker for interstitial lung disease in patients with polymyositis and dermatomyositis. J Intern Med 2011, Sep 
23. [Epub ahead of print]

75. -Gono T, Kawaguchi Y, Hara M, et al.: Increased ferritin predicts development and severity of acute interstitial lung disease as a complication of dermatomyositis. Rheumatology (Oxford) 2010, 49:1354-1360. Significantly high levels of serum ferritin were observed in DM patients with acute or subacute ILD, and patients with ferritin more than $1500 \mathrm{ng} / \mathrm{ml}$ showed poor prognosis.

76. Gono T, Kawaguchi Y, Ozeki E, et al.: Serum ferritin correlates with activity of anti-MDA5 antibody-associated acute interstitial lung disease as a complication of dermatomyositis. Mod Rheumatol 2011, 21:223-227.

77. Stone KB, Oddis CV, Fertig N, et al.: Anti-Jo-1 antibody levels correlate with disease activity in idiopathic inflammatory myopathy. Arthritis Rheum 2007, 56:3125-3131.

78. Sato S, Kuwana M, Fujita T, et al.: Amyopathic dermatomyositis developing rapidly progressive interstitial lung disease with elevation of anti-CADM-140/MDA5 autoantibodies. Mod Rheumatol 2011, Nov 29. [Epub ahead of print]

79. Nawata Y, Kurasawa K, Takabayashi K, et al.: Corticosteroid resistant interstitial pneumonitis in dermatomyositis/polymyositis: prediction and treatment with cyclosporine. J Rheumatol 1999, 26: 1527-1533.

80. Shinohara T, Hidaka T, Matsuki Y, et al.: Rapidly progressive interstitial lung disease associated with dermatomyositis responding to intravenous cyclophosphamide pulse therapy. Intern Med 1997, 36: 519-523.

81. Yoshida T, Koga H, Saitoh F, et al.: Pulse intravenous cyclophosphamide treatment for steroid-resistant interstitial pneumonitis associated with polymyositis. Intern Med 1999, 38: 733-738.

82. Yamasaki Y, Yamada H, Yamasaki M, et al.: Intravenous cyclophosphamide therapy for progressive interstitial pneumonia in patients with polymyositis/dermatomyositis. Rheumatology (Oxford) 2007, 46: 124-130.

83. Tanaka F, Origuchi T, Migita K, et al.: Successful combined therapy of cyclophosphamide and cyclosporine for acute exacerbated interstitial pneumonia associated with dermatomyositis. Intern Med 2000, 39: 428-430.

84. Kameda H, Nagasawa H, Ogawa H, et al.: Combination therapy with corticosteroids, cyclosporin A, and intravenous pulse cyclophosphamide for acute/subacute interstitial pneumonia in patients with dermatomyositis. J Rheumatol 2005, 32: 1719-1726.

85. Maeda, K., Kimura, R., Komuta, K.Igarashi, T., Cyclosporine treatment for polymyositis/dermatomyositis: is it possible to rescue the deteriorating cases with interstitial pneumonitis? Scand J Rheumatol 1997, 26:24-29.

86. Takada K, Nagasaka K, Miyasaka N: Polymyositis/dermatomyositis and interstitial lung disease: a new therapeutic approach with T-cell-specific immunosuppressants. Autoimmunity 2005, 38:383-392.

87. Kotani T, Makino S, Takeuchi T, et al. Early intervention with corticosteroids and cyclosporin A and 2-hour postdose blood concentration monitoring improves the 
prognosis of acute/subacute interstitial pneumonia in dermatomyositis. J Rheumatol 2008, 35:254-259.

88. - Kotani T, Takeuchi T, Makino S, et al.: Combination with corticosteroids and cyclosporin-A improves pulmonary function test results and chest HRCT findings in dermatomyositis patients with acute/subacute interstitial pneumonia. Clin Rheumatol 2011, 30:1021-1028. The efficacy of combination with CS and CSP was reported in 14 DM patients with acute or subacute ILD.

89. -Nagai K, Takeuchi T, Kotani T, et al.: Therapeutic drug monitoring of cyclosporine microemulsion in interstitial pneumonia with dermatomyositis. Mod Rheumatol 2011, 21:32-36. CSP blood level, especially $C O$ and C2, is useful to monitor clinical and adverse effects of CSP, and once daily preprandial administration is beneficial in DM with progressive ILD.

90. Ochi S, Nanki T, Takada K, et al.: Favorable outcomes with tacrolimus in two patients with refractory interstitial lung disease associated with polymyositis/dermatomyositis. Clin Exp Rheumatol 2005, 23:707-710.

91. Oddis CV, Sciurba FC, Elmagd KA, et al.: Tacrolimus in refractory polymyositis with interstitial lung disease. Lancet 1999, 353: 1762-1763.

92. Wilkes MR, Sereika SM, Fertig N, et al.: Treatment of antisynthetase-associated interstitial lung disease with tacrolimus. Arthritis Rheum 2005, 52: 2439-2446.

93. -Sem M, Molberg O, Lund MB, et al.: Rituximab treatment of the anti-synthetase syndrome: a retrospective case series. Rheumatology (Oxford) 2009, 48:968-971. 11 refractory ILD patients with anti-synthetase syndrome were treated with rituximab, and 7 of 11 showed a short-term beneficial effect on ILD.

94. Vandenbroucke E, Grutters JC, Altenburg J, et al.: Rituximab in life threatening antisynthetase syndrome. Rheumatol Int 2009, 29:1499-1502.

95. Ball EM, Savage EM, Pendleton A: Refractory anti-synthetase syndrome treated with rituximab. Rheumatology (Oxford) 2010, 49:1013.

96. @Saketkoo LA, Espinoza LR: Experience of mycophenolate mofetil in 10 patients with autoimmune-related interstitial lung disease demonstrates promising effects. Am J Med Sci 2009, 337:329-335. MMF was administered to 10 patients with autoimmune-related ILD (Including 2 PM), and all patients showed stabilization and/or improvement of the disease.

97. - Morganroth PA, Kreider ME, Werth VP: Mycophenolate mofetil for interstitial lung disease in dermatomyositis. Arthritis Care Res (Hoboken) 2010, 62:1496-501. 3 out of 4 patients with DM-ILD who received MMF experienced improvement of pulmonary function and dyspnea and reduction of $C S$ doses.

98. Suzuki Y, Hayakawa H, Miwa S, et al.: Intravenous immunoglobulin therapy for refractory interstitial lung disease associated with polymyositis/dermatomyositis. Lung 2009, 187:201-206.

99. Dasrmalchi M, Grundtman C, Alexanderson H, et al.: A high incidence of disease flares 
in an open pilot study of infliximab in patients with refractory inflammatory myopathies. Ann Rheum Dis 2008, 67:1670-1677.

100. The Muscle Study Group: A randomized, pilot trial of etanercept in dermatomyositis. Ann Neurol 2011, 70:427-436.

101. Narazaki M, Hagihara K, Shima Y, et al.: Therapeutic effect of tocilizumab on two patients with polymyositis. Rheumatology (Oxford) 2011, 50:1344-1346.

102. Furlan A, Botsios C, Ruffatti A, et al.: Antisynthetase syndrome with refractory polyarthritis and fever successfully treated with the IL-1 receptor antagonist, anakinra: A case report. Joint Bone Spine_2008, 75:366-367. 
Table. Two subsets of ILD in myositis defined by autoantibodies

\begin{tabular}{|c|c|c|}
\hline & Anti-synthetase-related ILD & Anti-CADM-140-related ILD \\
\hline Target antigen & $\begin{array}{c}\text { aminoacyl-tRNA synthetases } \\
\text { (8 types) }\end{array}$ & MDA5/IFIH 1 \\
\hline Frequency in myositis & $\sim 30 \%$ in whole $\mathrm{PM} / \mathrm{DM}$ & $\begin{array}{l}\sim 10-20 \% \text { in } \mathrm{DM} \\
\sim 50-70 \% \text { in } \mathrm{C}-\mathrm{ADM}\end{array}$ \\
\hline Frequency of ILD & $70-95 \%$ & $50-90 \%$ \\
\hline Type of ILD & chronic or subacute & acute progressive \\
\hline Histopathology & $\begin{array}{l}\text { mostly NSIP } \\
\text { sometimes UIP and OP }\end{array}$ & $\begin{array}{l}\text { NSIP? (early stage) } \\
\text { DAD (end stage) }\end{array}$ \\
\hline HRCT pattern & $\begin{array}{l}\text { basilar GGO } \\
\text { reticular or linear opacity } \\
\text { rare honeycombing }\end{array}$ & $\begin{array}{l}\text { lower and random GGO/ } \\
\text { consolidation }\end{array}$ \\
\hline Prognosis & fair but recurrent & poor \\
\hline Response to treatment & fair & poor \\
\hline Recommended therapy & $\mathrm{GC}+\mathrm{CSP}$ or $\mathrm{TAC}$ & GC+IVCYC+CSP \\
\hline
\end{tabular}



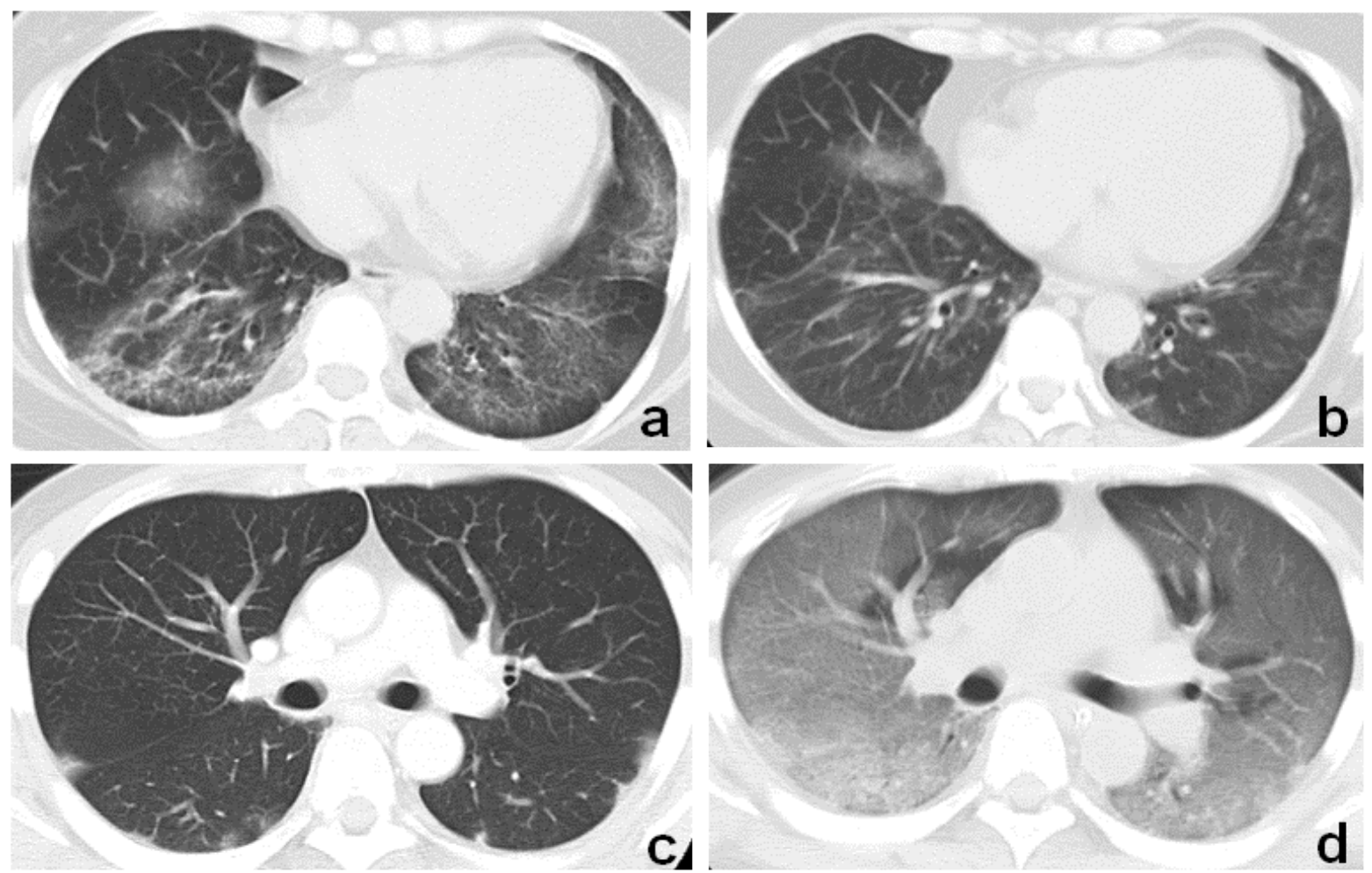

Figure. Radiographic imaging of CT in patients with myositis-interstitial lung disease having antisynthetase ( $a$ and $b$ ) and anti-CADM-140 antibody (c and d). A typical CT image shows basilar ground glass opacities (GGOs), reticular opacities, and traction bronchiectasis in a 47-year-old woman with dermatomyositis (DM) and anti-PL-7 antibody (a). A CT image of the same patient 1 year later shows significant improvement achieved by treatment with high-dose corticosteroids (b). CT image shows random consolidation/GGOs in subpleural region in a 44-year-old man with DM and anti-CADM-140 antibody at diagnosis (c). One month later, the patient developed severe respiratory failure and died despite treatment. Diffuse GGOs and consolidation were extended in the whole lung (d). 